\title{
Analysis of MYO-Inositol effect on spermatozoa motility, in hyper viscous ejaculates and in patients with grades II and III varicocele
}

\author{
Filomena Scarselli ${ }^{1}$, Anna Maria Lobascio ${ }^{1}$, Mario Terribile ${ }^{1}$, Valentina Casciani ${ }^{1}$, \\ Pierfrancesco Greco ${ }^{1}$, Giorgio Franco ${ }^{2}$, Maria Giulia Minasi ${ }^{1}$, Ermanno Greco ${ }^{1}$ \\ ${ }^{1}$ Centre for Reproductive Medicine, European Hospital, Rome, Italy; \\ ${ }^{2}$ Dept. Gynaecological-Obstetrical and Urological Sciences, Sapienza University, Rome, Italy.
}

\begin{abstract}
Summary The goal of this study is to evaluate MYOInositol effects on spermatozoa motility, in patients' ejaculates with severe varicocele or hyper viscosity. The study included normal viscosity ejaculate from 30 patients affected by varicocele and hyper viscosity ejaculate from 33 patients without any testicular pathologies. All selected samples showed sperm concentration $>2$ million $/ \mathrm{ml}$ and progressive motility $<32 \%$. In both groups, the pellet obtained after centrifugation in buffered medium, was divided in two aliquots, both incubated for 15 minutes at $37^{\circ} \mathrm{C}$ : one with MYO-Inositol and the other one, as control, only in phosphate buffered saline (PBS). Afterwards, the sperm progressive motility was assessed using Computer Assisted Sperm Analysis (CASA system).

Incubation with MYO-Inositol improved sperm progressive motility in high viscosity samples compared to control group $(38.9 \% \pm 3.0$ vs $24.35 \% \pm 2.41$, respectively; $p \leq 0.0001)$. Conversely, no statistically significant difference was observed in total sperm progressive motility in varicocele samples compared with control group $(22.7 \% \pm 2.07$ vs $26.7 \% \pm 3.31$, respectively; $p=0.085$ ).

The MYO-Inositol positive effect on spermatozoa motility may depend on the type of sperm damage: heavy structural and biochemical defects which typically affects patients with varicocele are not restored by Inositol. On the contrary, MYOInositol is able to improve sperm motility in semen samples with high viscosity, since those samples show no substantial structural sperm defects.
\end{abstract}

KEY WORDS: MYO-Inositol; Asthenozoospermia; Varicocele; Hyper-viscosity; Spermatozoa motility.

Submitted 14 July 2016; Accepted 19 August 2016

\section{INTRODUCTION}

Inositol $\left(\mathrm{C}_{6} \mathrm{H}^{12} \mathrm{O}_{6}\right)$ is a chemical compound existing in nine different stereoisomers synthesized from glucose-6phosphate. The most widespread form of Inositol in nature is MYO-Inositol (MYO-Ins), which belongs to the vitamin B group. MYO-Ins is synthesized by two different enzymes: MYO-1-phospate synthase (ISYNA1) and MYOmonophospatase-1 (IMPA1). These enzymes are present in high concentration in seminal plasma and in the testis, particularly in Sertoli cells rather than in germinal cells. After its synthesis, MYO-Ins is co-transported in the fluid of seminiferous tubules by the sodium/MYO-Inositol protein (SLC5A3), which is up-expressed in Sertoli cells $(1,2)$.

Several roles of MYO-Ins are known: it is involved in mitochondrial membrane potential (MMP) maintenance, in the control of intracellular $\mathrm{Ca}^{2+}$ concentration, in the cytoskeleton assembly, in gene expression regulation and it is also an important component of the structural lipids.

Phosphatidylinositol (PI) is involved in diacylglycerol (DAG) and Inositol triphosphate (IP3) formation, which are produced from phospholipase C (PLC) that hydrolyses phosphatidylinositol 4,5-bisphosphate (PIP2) to DAG and IP3, two second messengers involved in the cellular signal transduction and in the regulation of intracellular calcium concentration, respectively. IP3 binds to its receptor (IP3R) placed on the intracellular membrane of $\mathrm{Ca}^{2+}$ storages and triggers $\mathrm{Ca}^{2+}$ release necessary for sperm motility. There is evidence of the IP3Rlinked $\mathrm{Ca}^{2+}$ storages presence at the base of sperm flagellum, where only two membrane-bound organelles have been detected: mitochondria and the redundant nuclear envelope (RNE) (3). The latter arises from excess of nuclear envelope which is discarded during sperm nuclear chromatin condensation. Observations indicate that RNE has only a storages role and upon induction it releases $\mathrm{Ca}^{2+}$ which seems to act directly on the axoneme and, perhaps, indirectly on mitochondrial ATP production, both necessary to improve sperm motility. As reported in literature, MYO-Ins treatment does not affect the mitochondrial function of spermatozoa in normozoospermic patients, whereas it increases significantly the number of spermatozoa with high MMP in oligoasthenoteratospermic (OAT) patients (4). Observations show that the number of spermatozoa with high MMP levels correlates positively to sperm concentration and sperm progressive motility $(5,6)$. Furthermore spermatozoa with high MMP are directly linked to fertilization rate, in vivo and in vitro (7). However, the effects of MYO-Ins on phosphatidylserine (PS) externalization, an early marker of apoptosis, on chromatin compactness and on DNA fragmentation, in normozoospermic and OAT patients, were not known $(4,8)$.

Low sperm motility is a primary cause of male infertility,

No conflict of interest declared. 
associated with biochemical defects and/or with delayed or missing semen liquefaction. Semen samples coagulation and liquefaction with their physiological characteristics are still not fully understood; however, the negative effects of hyperviscosity on semen parameters, above all motility, are known. As reported in literature, hyperviscosity is due to a "trapping effect" that prevents normal sperm progression through the female genital tract ( 9 , 10-12). There is evidence of a correlation between hyperviscosity (SHV) and low levels of fructose in seminal plasma, due to an impaired function of the seminal vesicles (11). Some studies show a potential correlation between hyperviscosity and altered levels of $\mathrm{Ca}^{2+}$, zinc and acid phosphatase for prostate dysfunction $(13,14)$. Data reported in literature, correlate SHV to an excess of reactive oxygen species (ROS) (15), infections and/or genital tract's inflammations (16) and to genetic factors, such as the Cystic Fibrosis Transmembrane conductance Regulator (CFTR) gene expression. The cystic fibrosis causes defects in active ions transport $\left(\mathrm{Cl}^{-}\right)$, resulting in a lower water content in the intraluminal secretions that may be responsible for reduced semen fluidity and for progressive obstruction and destruction of the vas deferens and epididymis (17). Other authors demonstrate that hyper viscosity is not due to a single factor, but rather to several factors, which act in synergy (10).

Varicocele is a well-known infertile male condition. It is found in $10-20 \%$ of male population and it is associated to progressive decline of testicular function. Three grades of varicocele are known: grade I, palpable with Valsalva maneuver only; grade II, palpable without Valsalva maneuver and grade III, palpable and visible through the scrotal skin. Varicocele is associated to several conditions: altered testicular thermoregulation (18), blood stasis in spermatic veins (19) and elevated levels of abnormal sperm-associated Reactive Oxygen Species (ROS) (20). The effects of varicocele consist in a reduced testicular volume, a compromised sperm quality (motility, morphology and concentration) and reduced Leydig cells function. Some authors suggest a possible relation between varicocele and abnormal retention of sperm cytoplasmic droplets, typical of sub-fertile men (21).

The aim of this study was to evaluate the MYO-Ins effect on spermatozoa motility, in ejaculates of patients with severe varicocele (grade II and III) and in ejaculates with hyper viscosity.

\section{MATERIALS AND METHODS}

The study was performed from September 2012 to January 2013. Semen samples were collected by masturbation after 3-5 days of sexual abstinence. After 30 minutes of liquefaction at $37^{\circ} \mathrm{C}$, semen parameters were analysed according to World Health Organization (WHO) criteria 2010 (22). The thread length of both ejaculates with hyper viscosity and ejaculates of patients with grades II and III varicocele were measured, gently aspirating the semen liquid into a glass disposable pipette and leaving fall down, in gravity, a drop of sample on a slide. When the viscosity is normal, the semen sample leaves the pipette in small discrete drops; if viscosity is abnormal (increased), such as in SHV group, the drop will form a thread more than $2 \mathrm{~cm}$ long. The thread length was measured on a centimetre scale. Ejaculates of 30 patients (mean male age 39.9 years old, $\mathrm{SD} \pm 10.89$ ) with grade II and III varicocele (according to Sarteschi classification), with sperm concentration $>2$ million $/ \mathrm{ml}$, normal viscosity and low progressive motility $(<32 \%$, according to WHO 2010) (22), were included in the study (varicocele group).

Ejaculates of 33 patients without testicular pathologies (mean male age 39.6 years old, $\mathrm{SD} \pm 1.15$ ), with sperm concentration $>2$ million $/ \mathrm{ml}$, high viscosity (thread length $>2 \mathrm{~cm})$ and low progressive motility $(<32 \%$, according to WHO 2010) (22), were analysed (SHV group).

The enrolled semen samples were centrifuged in MOPSbuffered medium (G-MOPS TM PLUS - Vitrolife, Sweden) at $1800 \mathrm{rpm} / 10$ minutes an the pellet was divided in two aliquots: the first one was incubated with $2 \mathrm{mg} / \mathrm{ml}$ of MYO-Ins (Andrositol DGN, Lo.Li. Pharma S.R.L., Italy), for 15 minutes at $37^{\circ} \mathrm{C}$ temperature, in MOPS-buffered medium (G-MOPSTM PLUS - Vitrolife, Sweden) and the second one, as control, was incubated at the same conditions.

After incubation, sperm progressive motility, using Computer Assisted Sperm Analysis (CASA system - Sperm Class Analyzer Automatic Diagnostic Systems- MICROPTIC S.r.l. Spain), was evaluated.

\section{Statistical analysis}

Statistical analyses were performed using Student T-test and Wilcoxon T-test (parametric and non-parametric statistical test, respectively); P value of $<0.05$ was considered to be statistically significant. Results were expressed as mean $\pm \mathrm{SD}$.

\section{Results}

No statistically significant differences in total sperm progressive motility (slow and rapid) were observed in ejaculates of patients with grades II and III varicocele, after incubation with MYO-Ins and in control group $(22.7 \% \pm$ 2.07 and $26.7 \% \pm 3.31$, respectively; P student $=0.085$ ). On the contrary, incubation with MYO-Ins improved sperm progressive motility in SHV group. Our data demonstrate an enhanced sperm motility in semen with hyper viscosity $(38.9 \% \pm 3.0)$, compared to control group $(24.35 \% \pm 2.41)$ (Wilcoxon test $\mathrm{p} \leq 0.0001$; Figure 1).

The effects of MYO-Ins treatment observed in ejaculates of patients of varicocele group and in ejaculates of SHV group, have been separated in four categories, according to ANDROSITOL-LAB procedure, which divides the responses to Inositol in the following classes, summarized in Table 1:

1. High responder (HR): samples with increased sperm progressive motility from $\geq 60 \%$ to $100 \%$.

2. Middle responder (MR): samples with increased sperm progressive motility from $\geq 30 \%$ to $<60 \%$.

3. Poor responder (PR): samples with increased sperm progressive motility from $\geq 1 \%$ to $<30 \%$.

4. No responder (NR): samples with none increased sperm progressive motility. 


\section{Figure 1.}
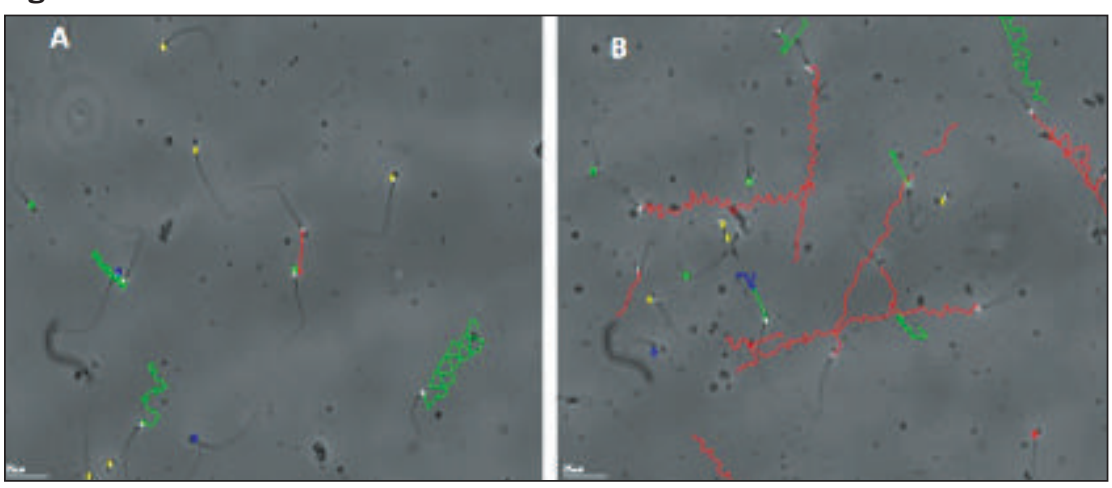

Analysis of image, using CASA system, of spermatozoa motility in ejaculates with hyper viscosity not incubated with MYO-Inositol (control, part A) and in ejaculates with hyper viscosity incubated with MYO-Inositol (part B). The coloured lines indicate the spermatozoa showing rapid progressivity (red), slow progressivity (green), in-situ motility (blue) and immotile spermatozoa (yellow), respectively. In hyper viscosity samples, the lines red and green are significantly increased after incubation with MYO-Inositol.

Table 1.

\begin{tabular}{|lcccc|}
\hline & $\begin{array}{c}\text { HR } \\
(\geq \mathbf{6 0}-\mathbf{1 0 0}) \%\end{array}$ & $\begin{array}{c}\text { MR } \\
(\geq \mathbf{3 0}-<\mathbf{1 0 0}) \%\end{array}$ & $\begin{array}{c}\text { PR } \\
(\geq \mathbf{1}-<\mathbf{3 0}) \%\end{array}$ & $\begin{array}{c}\text { NR } \\
(\mathbf{0}) \%\end{array}$ \\
\hline SHV group & $19 / 33(57.6 \%)$ & $8 / 33(24.2 \%)$ & $4 / 33(12.1 \%)$ & $2 / 33(6.1 \%)$ \\
\hline Varicocele group & $6 / 30(20 \%)$ & $5 / 30(16.7 \%)$ & $8 / 30(26.7 \%)$ & $11 / 30(36.7 \%)$ \\
\hline
\end{tabular}

Effect of MYO-Inositol on progressive sperm motility in the ejaculates of patients with hyperviscosity or with varicocele $(H R=$ High Responder, $M R=$ Middle Responder , $P R=$ Poor Responder, NR $=$ No Responder).

\section{Discussion}

The aim of this study was to evaluate the MYO-Ins effects on sperm motility in samples with SHV or varicocele, in which asthenozoospermia is a common features. A positive effect of Inositol on sperm progressive motility (slow and rapid) in ejaculates of patients with SHV was observed. Particularly, sperm progressive motility increased from $\geq$ $30 \%$ to $<60 \%$ (MR) and from $\geq 60 \%$ to $100 \%$ (HR), in SHV group. An increase in sperm progressive motility from $\geq 1 \%$ to $<30 \%$ (PR) or absence of response (NR) to MYOIns was observed in varicocele group.

Asthenozoospermia is one of male infertility causes, that involves an array of functional and biochemical sperm defects. Asthenozoospermic patients show both low sperm motility and low fertilizing ability due to their poor capability of hyper activation-capacitation linked. A relationship between sperm membrane fluidity and tyrosine phosphorylation is well known. Specifically, a deficit in tyrosine phosphorylation in asthenozoospermic patients produces a low sperm membrane fluidity and, therefore, a low sperm motility (23).

Some Authors (24), found an increase in sperm motility after 2 hours of incubation with MYO-Ins ( $2 \mathrm{mg} / \mathrm{ml})$, in OAT samples. In our study, a positive Inositol effect, at the same concentration, is observed on motility in SHV samples, after 15 minutes of incubation. Incubation attempts at 15 minutes, 30 minutes and 2 hours were done, but the highest Inositol effect on semen samples was observed at 15 minutes.

The faster Inositol effect observed in this study, is probably due to the samples rinsing with MOPS-buffered medium, before MYO-Ins treatment. It is possible that in SHV samples, the most of mucoid mass in which spermatozoa are tangled is dissolved or taken-away by rinsing. As a consequence, MYO-Ins may easily tie up sperm and mitochondrial membrane and active ion fluxes (mainly $\mathrm{HCO}^{3-}, \mathrm{Na}^{+}, \mathrm{K}^{+}$, and $\mathrm{Ca}^{2+}$ ) which are involved in tyrosine phosphorylation and in sperm capacitation (motility and hyperactivation) (25).

However, our hypothesis is in agree with Colone et al. (24), who observed, through Scansion Electron Microscopy (SEM), that spermatozoa of OAT patients appear entirely covered with an amorphous fibrous material, perhaps responsible of semen high viscosity and consequently of a reduced sperm motility. Additionally, Colone observed, through Transmission Electron Microscopy (TEM), altered mitochondrial cristae, placed in sperm intermediate tract of OAT samples. Nevertheless, after incubation with MYO-Ins, the authors found that, the surface spermatozoa and mitochondrial cristae of enrolled samples, appeared to be more similar to the controls (absence of amorphous fibrous material on sperm surface and intact mitochondrial cristae). These results show the ability of Inositol to dissolve the amorphous fibrous material and to improve spermatozoa motility, perhaps inducing a modification in semen samples $\mathrm{PH}$ and then acting on spermatozoa plasma and mitochondrial membranes.

Even the ejaculates of patients with grade II and III varicocele show low sperm motility. Nevertheless, in this study, the incubation with MYO-Ins did not always increase progressive sperm motility as in SHV samples. It is possible that $\mathrm{PR}$ and NR, observed in varicocele group, are due to a damaged spermatogenesis and/or to irreversibly altered biochemical factors.

Several studies demonstrate that in varicocele, the secretions of impaired sex accessory glands, such as fructose, might have a negative influence on motility of ejaculated spermatozoa (26). Other studies assert that fructose levels in varicocele samples are not significantly different to those of samples without varicocele (27). Steroid hormones, such as testosterone, play a fundamental role not only in sexual differentiation and in expression of secondary sexual characteristics but also, in spermatogenesis. Wang et al. (28), report that the action of androgens is mediated by androgen receptors (AR), which act as a ligand-inducible transcription factor. AR ligands are: testosterone (T) and dihydrotestosterone (DHT), its more active metabolite. Ejaculated mammalian spermatozoa express AR in the head and in the mid piece, this 
latter corresponding to the mitochondria site. AR play an important role in sperm survival and capacitation, acting on PI3K/AKT signaling pathway (29-31). As reported, during capacitation, spermatozoa undergo a final maturation by increasing cholesterol efflux which acts on tyrosine phosphorylation and on sperm membrane fluidity (32). Some Authors (33), found that the basal cholesterol efflux is reduced in spermatozoa of patients with varicocele compared to spermatozoa of patients without varicocele. This provokes a reduced sperm membrane fluidity and thus a decreased motility, in varicocele samples. These authors also observed that in normal spermatozoa, DHT induced G6PDH activity, an enzyme involved in glucose metabolism, through PI3K/AKT pathway. This effect is not observed in varicocele samples (33). Jackaman et al. (34) and Al-Daghistani et al. (27) demonstrate that testosterone levels in samples without varicocele are lower than in samples with varicocele. This feature may indicate severe atrophy of seminiferous tubules, sclerosis and Leydig cells hyperplasia, causing the humoral immune response and then ASA (Anti Sperm Antibodies) production; these latter, as known, are involved in a reduced sperm motility. It is probable that in ejaculates with varicocele, oxidative stress and spermatogenesis dysfunction are the two main causes of an impaired tyrosine phosphorylation and, consequently, an altered membrane fluidity. It is known, for example, that sperm motility and capacitation are related to sperm membrane composition: high levels of polyunsaturated fatty acids makes it oversensitive to the attack of reactive oxygen species which cause both an altered tyrosine phosphorylation and altered signal transduction, resulting in low membrane fluidity and consequently, reduced sperm motility $(35,36)$.

In sustain of that, some authors establish that spermatozoa of patients with varicocele, have an abnormal retention of cytoplasmic droplets due to excessive ROS production (21). ROS are negatively correlated with sperm motility, through oxidation of membrane fatty acids which alter sperm membrane fluidity $(37,38)$. Excessive ROS production, associated to increased testicular temperature, typical condition of varicocele, and to abnormal retention of cytoplasmic droplets, due to an impaired spermatogenesis, causes a reduced/altered sperm functions correlated with DNA damages. Studies confirm higher levels of damaged and fragmented DNA ROS related and thus reduced MMP and decreased sperm motility (39), in infertile men with varicocele compared to fertile men without varicocele (40-41).

\section{Conclusions}

The lack or absence of MYO-Inositol effect observed in varicocele group seems principally due to impaired spermatogenesis and to heavy biochemical and structural sperm defects. Defects linked to varicocele considerably compromise sperm functionality on which Inositol has no or partially positive effect (the benefits of varicocelectomy to improve sperm quality are discussed). On the contrary the increase of sperm motility observed in SHV group after incubation with MYO-Inositol, may be connected to the absence of substantial structural sperm defects. The low spermatozoa motility observed in SHV samples is most probably related above all to mucoid mass in which sperm are tangled and of which, the partial or total removal, allows the improving of sperm motility by Inositol activity.

In conclusion MYO-Inositol appears to have a positive effect on spermatozoa motility, depending on the type of sperm damage: heavy structural defects, typical of varicocele samples, are not restored by Inositol.

\section{REFERENCES}

1. Robinson R, Fritz IB. Myoinositol biosynthesis by Sertoli cells, and levels of myoinositol biosynthetic enzymes in testis and epididymis. Can J Biochem. 1979; 57:962-7.

2. Chauvin TR, Griswold MD. Characterization of the expression and regulation of genes necessary for myo-inositol biosynthesis and transport in the seminiferous epithelium. Biol. Reprod. 2004; 701:744-51.

3. Ho HC, Suarez SS. Characterization of the intracellular calcium store at the base of the sperm flagellum that regulates hyperactivated motility. Biol Reprod. 2003; 68:1590-6.

4. Condorelli RA, La Vignera S, Di Bari F, et al. Effects of myoinositol on sperm mitochondrial function in-vitro. Eur Rev Med Pharmacol Sci. 2011; 15:129-134.

5. Wang X, Sharma RK, Gupta A, et al. Alterations in mitochondria membrane potential and oxidative stress in infertile men: a prospective observational study. Fertil Steril. 2003; 80 Suppl 2:844-50.

6. Lobascio AM, De Felici M, Anibaldi M, et al. Involvement of seminal leukocytes, reactive oxygen species, and sperm mitochondrial membrane potential in the DNA damage of the human spermatozoa. Andrology 2015; .3:265-70.

7. Marchetti C, Obert G, Deffosez A, et al. Study of mitochondrial membrane potential, reactive oxygen species, DNA fragmentation and cell viability by flow cytometry in human sperm. Hum Reprod. 2002; 17:1257-65.

8. Condorelli RA, La Vignera S, Bellanca S, et al. Myoinositol: Does it improve sperm mitochondrial function and sperm motility? Urology. 2012; 79:1290-5.

9. Elzanaty S, Malm J, Giwercman A. Visco-elasticity of seminal fluid in relation to the epididymal and accessory sex gland function and its impact on sperm motility. Int J Androl. 2004; 27:94-100.

10. Elia J, Delfino M, Imbrogno $N$, et al. Human semen hyperviscosity: prevalence, pathogenesis and therapeutic aspects. Asian J Androl. 2009; 11:609-615.

11. Gonzales GF, Kortebani G, Mazzolli AB. Hyperviscosity and hypofunction of the seminal vesicles. Arch Androl. 1993; 30:63-68.

12. Siciliano L, Tarantino P, Longobardi F, et al. Impaired seminal antioxidant capacity in human semen with hyperviscosity or oligoasthenozoospermia. J Androl. 2001; 22:798-803.

13. Andrade-Rocha FT. Physical analysis of ejaculate to evaluate the secretory activity of the seminal vesicles and prostate. Clin Chem Lab Med. 2005; 43:1203-10.

14. Carpino A, Siciliano L. Unaltered protein pattern/genital tract secretion marker levels in seminal plasma of highly viscous human ejaculates. Arch Androl. 1998; 41:31-5.

15. Aydemir B, Onaran I, Kiziler AR, et al. The influence of oxidative damage on viscosity of seminal fluid in infertile men. J Androl. 2008; 29:41-6. 
16. Henkel R, Maass G, Jung A, et al. Age-related changes in seminal polymorphonuclear elastase in men with asymptomatic inflammation of the genital tract. Asian J Androl. 2007; 9:299-304.

17. Rossi T, Grandoni F, Mazzilli F, et al. High frequency of (TG) $m$ Tn variant tracts in the cystic fibrosis transmembrane conductance regulator gene in men with high semen viscosity. Fertil Steril. 2004; 82:1316-22.

18. Zorgniotti AW, Macleod J. Studies in temperature, human semen quality, and varicocele. Fertil Steril.1973; 24: 854-63.

19. Shafik A, Bedeir GA. Venous tension patterns in cord veins. I. In normal and varicocele individuals. J Urol. 1980; 123:383-5.

20. Hendin BN, Kolettis PN, Sharma RK, et al. Varicocele is associated with elevated spermatozoa reactive oxygen species production and diminished seminal plasma antioxidant capacity. J Urol.1999; 161:1831-4.

21. Zini A, Defreitas G, Freeman M, et al. Varicocele is associated with abnormal retention of cytoplasmic droplets by human spermatozoa. Fertil Steril. 2000; 74:461-4.

22. WHO Cooper TG, et al. World Health organization reference values for human semen characteristics. Human Reproduction Update 2010; 16:231-245.

23. Buffone MG, Calamera JC, Verstraeten SV, Doncel GF. Capacitation-associated protein tyrosine phosphorylation and membrane fluidity changes are impaired in the spermatozoa of asthenozoospermic patients. Reproduction. 2005; 129:697-705.

24. Colone M, Marelli G, Unfer V, et al. Inositol activity in oligoasthenoteratospermia-An in vitro study. Eur Rev Med Pharmacol Sci. 2010; 14:891-6.

25. Visconti PE, Kopf GS. Regulation of protein phosphorylation during sperm capacitation. Biology of Reproduction. 1998; 59:1-6.

26. Andò S, Carpino A, Buffone M, et al. Fructose, prostatic acid phosphatase and zinc levels in the seminal plasma of varicoceles. International Journal of Fertility, 1990; 35, pp. 249-252.

27. Al-Daghistani HI, Hamad AW, Abdel-Dayem M, Aet al. Evaluation of Serum Testosterone, Progesterone, Seminal Antisperm Antibody, and Fructose Levels among Jordanian Males with a History of Infertility. Biochem Res Int. 2010; 409640.

28. Wang RS, Yeh S, Tzeng CR \& Chang C. Androgen receptor roles in spermatogenesis and fertility: lessons from testicular cell-specific androgen receptor knockout mice. Endocr Rev. 2009; 30:119-32.

29. Solakidi S, Psarra AM, Nikolaropoulos S, Sekeris CE. Estrogen receptors $a$ and $b$ (ERa and $E R b)$ and androgen receptor (AR) in human sperm: localization of $E R b$ and $A R$ in mitochondria of the midpiece. Hum Reprod. 2005; 20:3481-7.

30. Aquila S, Middea E, Catalano S, et al. Human sperm express a functional androgen receptor: effects on PI3K/AKT pathway. Hum Reprod. 2007; 22:2594-605.

31. Zalata AA, Mokhtar N, Badawy Ael-N, et al. Androgen receptor expression relationship with semen variables in infertile men with varicocele. J Urol. 2013; 189:2243-7.

32. Cross NL. Role of cholesterol in sperm capacitation. Biol Reprod. 1998; 59:7-11.

33. Guido C, Santoro M, De Amicis F, et al. Human sperm anatomy and endocrinology in varicocele: role of androgen receptor. Reproduction. 2014; 147:589-98.

34. Jackaman R, Ghanadian R, Ansell ID. Relationships between spermatogenesis and serum hormone levels in subfertile men. British
Journal of Obstetrics and Gynaecology. 1977; vol. 84, no. 9, pp. 692-696.

35. Aitken RJ, Harkiss D, Knox W, et al. A novel signal transduction cascade in capacitating human spermatozoa characterised by a redox-regulated, cAMP-mediate induction of tyrosine phosphorylation. J Cell Sci. 1998; 111:(Pt 5):645-56.

36. Calamera J, Buffone M, Ollero $M$, et al. Superoxide dismutase content and fatty acid composition in subsets of human spermatozoa from normozoospermic, asthenozoospermic, and polyzoospermic semen samples. Mol Reprod Dev. 2003; 66:422-30.

37. Gomez E, Buckingham DW, Brindle J, et al. Development of an image analysis system to monitor the retention of residual cytoplasm by human spermatozoa: correlation with biochemical markers of the cytoplasmic space, oxidative stress, and sperm function. J Androl. 1996; 17:276-87.

38. Keating J, Grundy CE, Fivey PS, et al. Investigation into the association between the presence of cytoplasmic residues on the human sperm midpiece and defective sperm function. J Reprod Fertil. 1997; 110:71-7.

39. Blumer CG, Fariello RM, Restelli AE, et al. Sperm nuclear DNA fragmentation and mitochondrial activity in men with varicocele. Fertil Steril. 2008; 90:1716-22.

40. Saleh RA, Agarwal A, Sharma RK, et al. Evaluation of nuclear DNA damage in spermatozoa from infertile men with varicocele. Fertil Steril. 2003; 80:1431-6.

41. Smith R, Kaune H, Parodi D, et al. Increased sperm DNA damage in patients with varicocele: relationship with seminal oxidative stress. Hum Reprod. 2006; 21:986-93.

\section{Correspondence}

Filomena Scarselli, MSc (Corresponding Author)

filomenascarselli@virgilio.it

Anna Maria Lobascio, PhD

Mario Terribile, $\mathrm{PhD}$

Valentina Casciani, PhD

Pierfrancesco Greco

Maria Giulia Minasi, MSc

Ermanno Greco, MD

Centre For Reproductive Medicine, European Hospital

Via Portuense 700, 00149 Rome, Italy

Giorgio Franco, MD

Dept. Gynaecological-Obstetrical and Urological Sciences,

Sapienza University, Rome, Italy 